\title{
Optical-CDMA in InP
}

\author{
Ronald G. Broeke, Jin Cao, Chen Ji, Sang-Woo Seo, Member, IEEE, Yixue Du, Student Member, IEEE, \\ Nick K. Fontaine, Jong-Hwa Baek, John Yan, Francisco M. Soares, Fredrik Olsson, \\ S. Lourdudoss, Senior Member, IEEE, Anh-Vu H. Pham, Senior Member, IEEE, Michael Shearn, \\ Axel Scherer, and S. J. Ben Yoo, Senior Member, IEEE
}

\begin{abstract}
This paper describes the InP platforms for photonic integration and the development on these platforms of an optical code division multiple access (O-CDMA) system for local area networks. We demonstrate three building blocks of this system: an optical pulse source, an encoder/decoder pair, and a threshold detector. The optical pulse source consists of an integrated colliding pulse-mode laser with nearly transform-limited $10 \mathrm{~Gb} / \mathrm{s}$ pulses and optical injection locking to an external clock for synchronization. The encoder/decoder pair is based on arrayed waveguide gratings. Bit-error-rate measurements involving six users at $10 \mathrm{~Gb} / \mathrm{s}$ showed error-free transmission, while O-CDMA codes were calibrated using frequency resolved optical gating. For threshold detection after the decoder, we compared two Mach-Zehnder interferometer (MZI)-based optical thresholding schemes and present results on a new type of electroabsorber-based MZI.
\end{abstract}

Index Terms-Arrayed-waveguide gratings (AWG), crosscorrelation frequency-resolved optical gating (XFROG), InP platform, Mach-Zehnder interferometer (MZI), optical code division multiple access (O-CDMA), optical thresholding, photonic integrated circuit.

\section{INTRODUCTION}

$\mathbf{P}$ HOTONIC integration has been maturing to a level where system scale functionality can be integrated on a single substrate. Most suitable for advanced photonic integration is the InGaAsP/InP material system, because of its tunable bandgap across the 1.3- and 1.5- $\mu \mathrm{m}$ wavelength communication windows of optical fiber and, therefore, the ability to integrate active and passive waveguides. An InP platform supporting active-passive integration provides three key functionalities on a single substrate: passive optical waveguiding (with bend radii down to 50-500 $\mu \mathrm{m}$ for compact design), phase shifting, and amplification/detection of light. Platforms usually add one or more functionalities like electroabsorption modulators, polarization converters, spot-size converters, on-chip mirrors, or/and electrical high-frequency (HF) phase and HF amplitude modulators. Several InP platforms, using different techniques, have been reported: quantum well $(\mathrm{QW})$ intermixing [1] enables activepassive integration without regrowth, but often at the expense

Manuscript received December 18, 2006; revised June 15, 2007. This work was supported in part by DARPA and SPAWAR under Agreement N66001-01-1 and under Contract HR0011-04-1-0054.

R. G. Broeke, J. Cao, C. Ji, S. W. Seo, Y. Du, N. K. Fontaine, J. H. Baek, J. Yan, F. Soares, A.-V. H. Pham, and S. J. B. Yoo are with the Electrical Engineering Department, University of California, Davis, CA 95616 USA (e-mail: yoo@ece.ucdavis.edu).

F. Olsson and S. Lourdudoss are with the School of Information and Communication Technology, Royal Institute of Technology, S-16440 Stockholm, Sweden.

M. Shearn and A. Scherer are with the Division of Engineering and Applied Science, California Institute of Technology, Pasadena, CA 91125 USA.

Digital Object Identifier 10.1109/JSTQE.2007.902854 of increased waveguide loss due to band-tail edge absorption and the introduction of lattice defects by the implanted ions. Butt-joint selective-area (SA) regrowth in combination with a double-etch technique [2], [3] provides flexibility in the positioning and type of the active layer and allows for compact design by high-contrast waveguide bends. However, it is difficult to control the regrowth of large-step butt-joints and to accurately control the etch depth required for the low-contrast waveguides. Integration by symmetric twin-waveguide structure [4] does not require a regrowth, but introduces possible optical absorption in the taper region. Also, the effective index of both waveguides should match accurately to limit the taper lengths.

In our approach [5], we use a hydride vapour phase epitaxy (HVPE) lateral regrowth for simultaneous waveguide passivation and planarization. It allows for a noncritical waveguide etching similar to buried waveguide processes. In this paper, we report on two platforms with respect to the development of an integrated O-CDMA system: one platform for low frequency (LF) components and a new platform for high frequency (HF). The additional tradeoffs in chip design introduced by HF components for rapid code reconfiguration justifies a separate HF platform. Furthermore, we reduced waveguide loss by improved HVPE lateral growth conditions for more uniform sidewall coverage. We employed frequency-resolved optical gating for more accurate optical-phase calibration of the O-CDMA coding/decoding and generating shorter pulses from the modelocked laser. Finally, we introduced a new Mach-Zehnder interferometer (MZI) thresholder design.

We develop the O-CDMA system using a parallel-track approach. On the first track, we realize a table-sized optical freespace/fiber-based system to demonstrate feasibility, principle of operation, and to lay the groundwork for system scale characterization. For example, the free-space SLM-based 32-user $\times$ $10 \mathrm{~Gb} / \mathrm{s}$ O-CDMA system [6]. On the other track, we develop the O-CDMA system on an InP platform. The parallel track approach provides an environment, in which we progress toward full integration by inserting InP devices incrementally into a proven operating system architecture. Full integration in semiconductor is a prerequisite for robust and large-scale practical deployment.

\section{INTEGRATION TECHNOLOGY}

In this section, we give a brief overview of the integrated waveguides in our two platforms: for LF and HF (10-40 GHz).

First, we consider the LF O-CDMA platform. Fig. 1 shows a fully integrated O-CMDA system consisting of a transmitter and receiver section. The transmitter consists of a mode-locked laser (MLL) integrated with a spectral-phase encoder. The receiver 


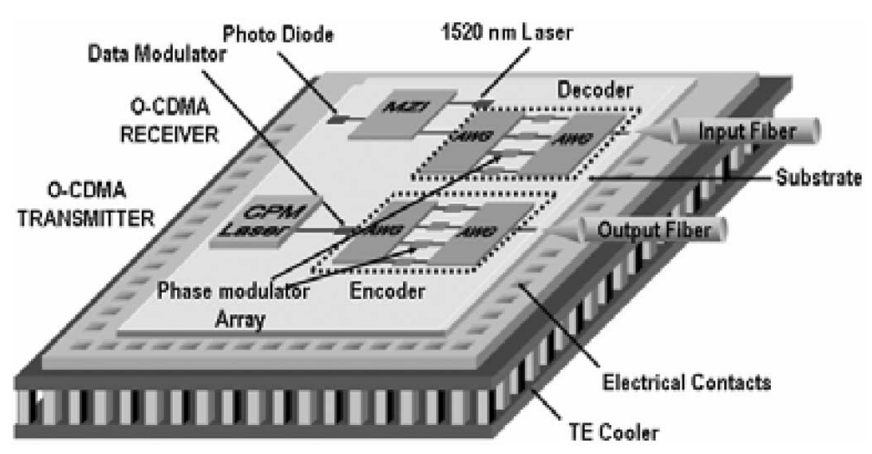

Fig. 1. Schematic picture of a fully integrated O-CDMA system.

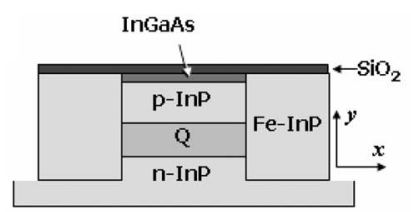

(a)

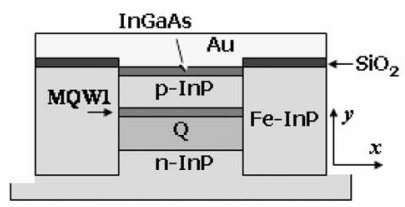

(c)

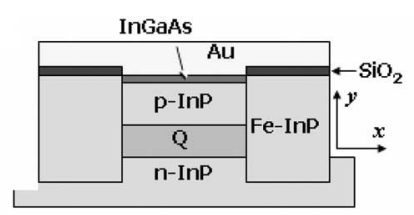

(b)

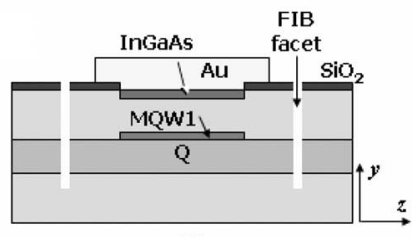

(d)

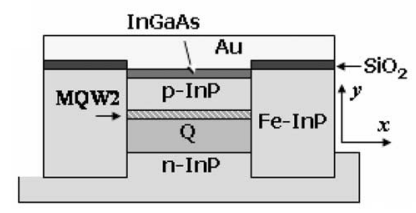

(e)

Fig. 2. Cross-sectional view of waveguides. (a) Passive waveguide. (b) Phase shifter. (c) SOA. (d) Laser with FIB facets. (e) EA.

consists of a decoder integrated with an MZI-based threshold detector (MZI-TD).

Fig. 2 shows the cross-sectional view of the waveguide types in the O-CDMA devices. The orientation of the cross sections is such that the waveguide widths are displayed in the $x$-direction (lateral), thickness in the $y$-direction, and the propagation direction of the guided light in the $z$-direction (longitudinal). Fig. 2(a) displays a passive waveguide of a 500-nm-thick quarternary $\mathrm{Q}(1.15)$ waveguiding (film) layer sandwiched in InP. Lateral regrown $\mathrm{Fe}-\mathrm{InP}$ layers passivate the sidewalls of the waveguide and provide electrical isolation. In addition, the lateral regrowth planarizes the waveguide for subsequent metallization steps. The typical waveguide width is $3 \mu \mathrm{m}$, which is a compromise between low propagation loss $(2.5 \mathrm{~dB} / \mathrm{cm})$ and single-mode waveguiding (avoiding the symmetrical second-order mode). Fig. 2(b) shows a phase shifter, whose refractive index can be changed by injecting carriers in the guiding layer (forward bias), or by applying an electrical field across the Q layer (reverse bias). The metal (AuGeNi/Au) and highly doped InGaAs layer on top provide a low-resistance n-contact for electrical connection to an outside current or voltage source, for instance, by wire

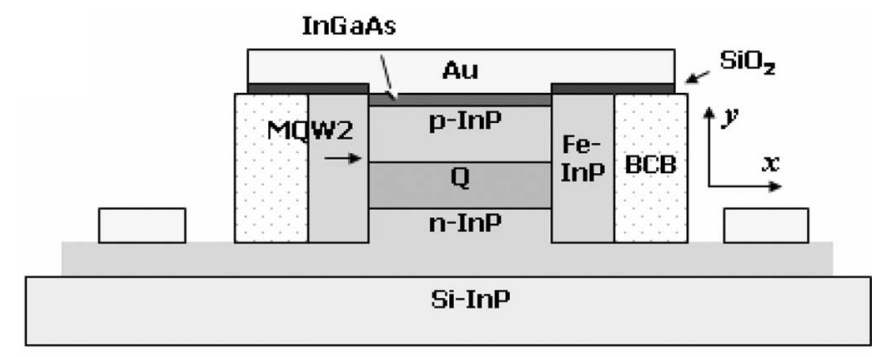

(a)

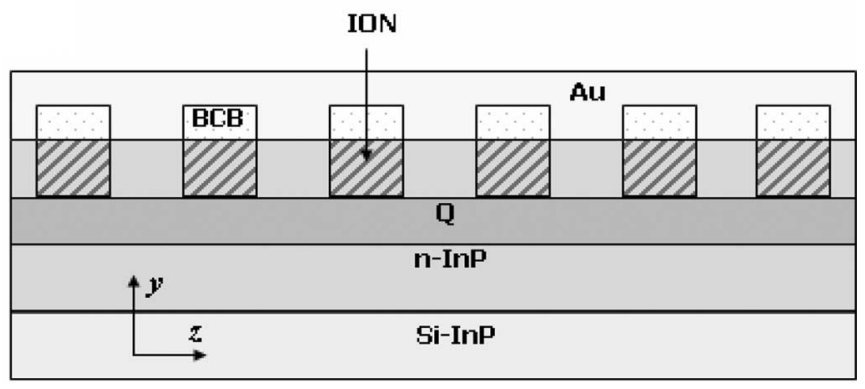

(b)

Fig. 3. Traveling-wave phase modulator. (a) Lateral cross section. (b) Longitudinal cross section.

bonding in packaged devices. Semiconductor optical amplifiers (SOA) [Fig. 2(c)] have multiquantum well (MQW) layer on top of the film layer (MQW1). The MQW1 consists of six wells of 7-nm-thick Q(1.25) InGaAsP and 6-nm-thick InGaAs barriers. The designed gain peak lies at $1550 \mathrm{~nm}$. Fig. 2(d) shows a longitudinal cross section of an SOA with etched facets. The focused ion beam (FIB) etched facets provide optical feedback for the SOA, and create a laser suitable for integration of the laser with other devices on a single substrate A second type of MQW layer, MQW2 [Fig. 2(e)] provides a peak gain at around $1490 \mathrm{~nm}$ and has been optimized for electroabsorption (EA) modulator operation; for instance, for data modulating a pulse train from a 10-GHz mode-locked laser, or for phase shifting in an MZI structure, as described later in this paper.

Fast code reconfiguration in the encoder/decoder demands HF traveling-wave phase modulators. These modulators need to be optimized for low propagation loss of HF electrical fields, velocity matching of the optical group and electrical phase velocity, and maximum overlap between the optical fields and the electrical field.

Fig. 3 shows the cross sections of a traveling wave modulator. The most pronounced change with respect to the stack from Fig. 1(a) is the replacement of the n-doped InP substrate with a semi-insulating (SI) substrate. The n-contacts are placed on the top side of the chip, on top of an n-InP buffer layer. This configuration reduces electrical propagation loss by reducing the amount of electrical field in $n$-doped layers. A benzocyclobutene (BCB) layer on both sides of the Fe-InP provides additional support for the metal contact layer on top. $\mathrm{The}^{\mathrm{SiO}_{2}}$ layers provide an electrically isolated cohesion layer for the metal. Removal of the n-buffer layer between adjacent modulators isolates their n-contacts. 


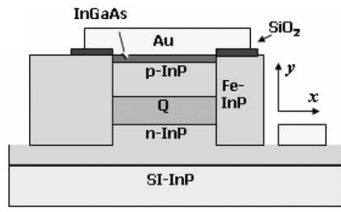

(a)

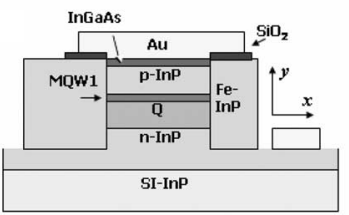

(c)

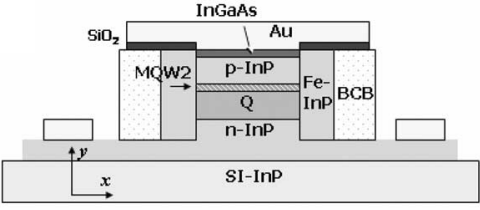

(b)

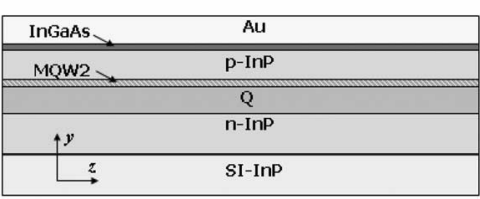

(d)
Fig. 4. Cross sections. (a) Phase shifter. (b) SOA. Cross sections of highfrequency EA modulator. (c) Lateral. (d) Longitudinal.

The epitaxy on an SI substrate consists of a 500-nm-thick n-InP buffer layer, a 500-nm Q (1.15) layer, a 2.0- $\mu \mathrm{m}$ p-InP layer with gradually increasing p-doping toward the top, and a 100-nm-thick InGaAs contact layer. A RIE etch through the Q layer defines the waveguides. A lateral Fe-InP regrowth passivates the waveguides and planarizes the surface. In order to reduce the overlap of the electrical field with the laterally regrown Fe-InP, a wet chemical etch reduces the Fe-InP width to $4 \mu \mathrm{m}$ on both sides, which is wide enough not to affect the optical guiding properties of the waveguide. Furthermore, since the microwave electrical field tends to have a lower propagation velocity compared to the optical field, increasing the electrical field confinement in air (by thinner Fe-InP) improves velocity matching. The etching stops at the top of the n-buffer layer to enable the n-contacts.

Fig. 3(b) shows the same modulator, but in a longitudinal cross section through the waveguide center. The segmentation of the p-contact geometry provides velocity matching of the electrical phase with the optical group velocity. The segmentation consists of ion-implantation to locally destroy the conductance of the $\mathrm{p}$ layer and a BCB layer to avoid metal-semiconductor contact on the ion-implanted segments.

Due to the SI substrate, all electrical contacts need to be placed at the top side of the device. Fig. 4(a) shows an LF phase shifter in the HF-platform and Fig. 4(b) shows an SOA. In case of current injection into the chip, the resistance from the $\mathrm{p}$ - to $\mathrm{n}$-contact should be minimized. The resistance of the n-buffer to the n-contact can be limited to a few ohms by placing the n-contact as close as possible to the waveguide and by making it sufficiently doped and thick. Fig. 4(c) and (d) displays an EA with high-speed electrical connections. It is similar to the traveling-wave modulator, except that the EA is shorter (up to $300-\mu \mathrm{m}$ long), which makes velocity matching much less critical. Hence, no ion implantation is required.

\section{O-CDMA ON INP}

A SPECTS O-CDMA system employs spectral encoding of ultrashort optical pulses from a mode-locked laser for data transmission. Spectral coding in the transmitter spreads pulses in time at the expense of peak power. The decoder only reconstructs the ultrashort high-peak-power pulse if the encoder code

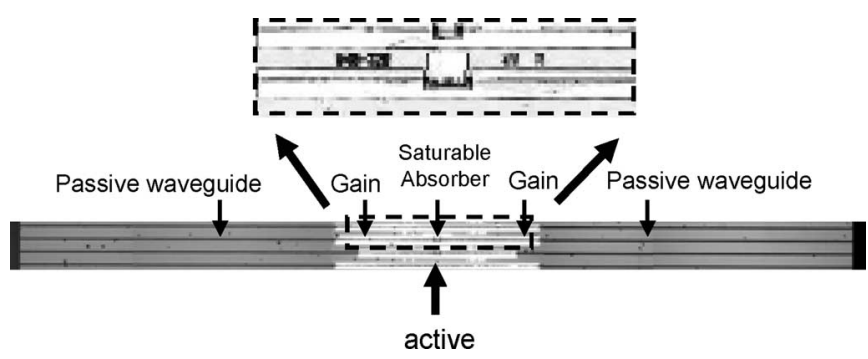

Fig. 5. SEM image of a 10-GHz CPM laser with integrated active-passive waveguides.

and decoder code are conjugates of each other. (The decoder and encoder are similar devices.) The receiver, after the decoder, discriminates correctly decoded pulses from incorrectly decoded pulses by nonlinear threshold detection. The receiver detects a " 1 " bit only if a pulse has sufficient peak power (correctly decoded). Otherwise, the receiver detects a " 0 " bit for a time-spread pulse. The following sections elaborate our results with these InP-based O-CDMA components.

\section{A. Mode-Locked Laser}

An ultrafast light source based on semiconductor modelocked (ML) laser is required as a transmitter in an O-CDMA integrated photonic circuit. We have developed a $10-\mathrm{GHz}$ colliding-pulse mode-locked laser (CPM) [8], [9] in the platform from Fig. 1, allowing for the monolithic integration of the CPM laser with other O-CDMA elements discussed in the remaining part of the paper. Compared to a regular mode-locked laser design, the CPM laser operates in the configuration where two symmetrically counter-propagating pulses collide and bleach the saturable absorber located at the center of the laser cavity, resulting in deeper saturation and more stable mode locking.

Fig. 5 shows the SEM image of a fabricated CPM laser. The required CPM cavity length for $10-\mathrm{GHz}$ operation exceeds $8 \mathrm{~mm}$, which will require a fairly high drive current for all allactive design. Furthermore, long $(>4 \mathrm{~mm}$ ) all-active-mode ML lasers for achieving lower repetition rate tend to suffer from strong pulse shaping effects, resulting in poor chirping and jitter performance [10]. Adopting the active-passive integration process in Fig. 1 allows for reducing the active section length to minimizing these effects and the drive current requirement [8]. The active-passive interfaces are designed to be laterally tilted at $45^{\circ}$ relative to the waveguide direction. This lateral tilt is critical for eliminating unwanted secondary pulses originating from residual reflections at the interfaces, resulting in much improved mode-locking performance compared to the untilted designs. The active region has a total length of $2000 \mu \mathrm{m}$, dividing into two gain sections and sandwiching the $45-\mu \mathrm{m}$-wide saturable absorber (SA) located at the center. The waveguide is further extended symmetrically on both sides with passive sections, forming the 8200- $\mu$ m-long laser cavity.

The CPM laser can be synchronized to an external system clock through either electrical hybrid mode locking (HML) [10], [11] at the fundamental or a subharmonic frequency, or through the optical synchronous mode locking (OSML) approach [12], [13]. In the electrical HML configuration, RF modulation signal 

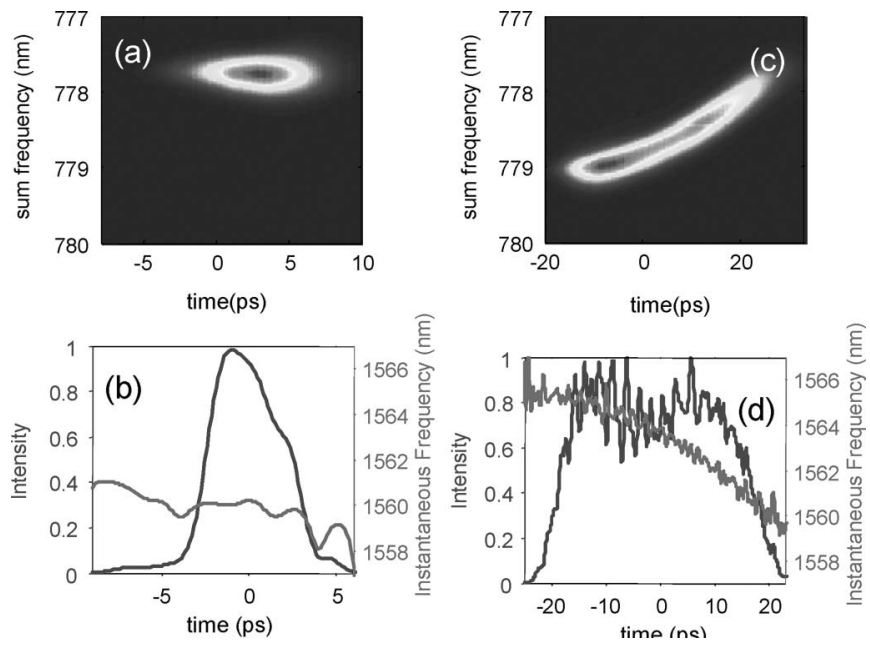

Fig. 6. (a) XFROG spectrogram and (b) extracted time-domain intensity and insantaneous frequency of a nearly transform-limited pulse. (c) XFROG spectrogram and (d) extracted time-domain intensity and instantaneous frequency of a highly chirped pulse from the same CPM laser under different biasing conditions.

is applied to the saturable absorber of the CPM laser through a ground-signal-ground microwave probe, while the two gain sections and the SA are dc-biased through dc needle probes (forward current injection) and the microwave probe (reverse bias voltage) through a bias-tee connection, respectively.

The CPM laser output pulse characteristics are very sensitive functions of the dc and RF biasing conditions. We utilized the cross-correlation frequency-resolved optical gating (XFROG) technique [14] (Fig. 9) for accurate extraction of the complete temporal amplitude and phase information, and the corresponding spectral domain profiles of the pulse simultaneously.

Fig. 6 shows the XFROG characterization of the CPM laser under two different bias conditions. In Fig. 6(a), with 89-mA gain section and $-7.5 \mathrm{~V} \mathrm{SA}$ biasing, the tightly distributed XFROG spectrogram indicates that the pulse is close to being transform limited. The extracted time-domain intensity and instantaneous frequency profiles in Fig. 6(b) indicates a pulse width of 5.7 ps with minimal frequency chirp, with timebandwidth product of 0.67 for a spectral width of $1.8 \mathrm{~nm}$. Fig. 6(c) shows the XFROG trace of the same device at a different biasing condition with $156-\mathrm{mA}$ gain section current, $-4.5 \mathrm{~V}$ across the SA. The extracted time-domain profile in Fig. 6(d) shows that the resulting pulse is over 40-ps wide, with both strong linear and higher order chirp components. The XFROG technique allows the full characterization and systematic and efficient optimization of all the individual biasing parameters of a CPM laser in real time.

We have previously reported in detail the electrical HML investigation of the 10-GHz CPM laser [8]. By applying the RF clock signal matching the passive ML frequency of $10.3 \mathrm{GHz}$ at $19 \mathrm{dBm}$, we obtained nearly transform-limited output with pulse width of 1.75 ps and time-bandwidth product 0.33 , with relatively low-timing jitter. This short-pulse low-chirp performance can be attributed to the careful balancing of the pulse broadening and chirping effects inside the relatively short gain

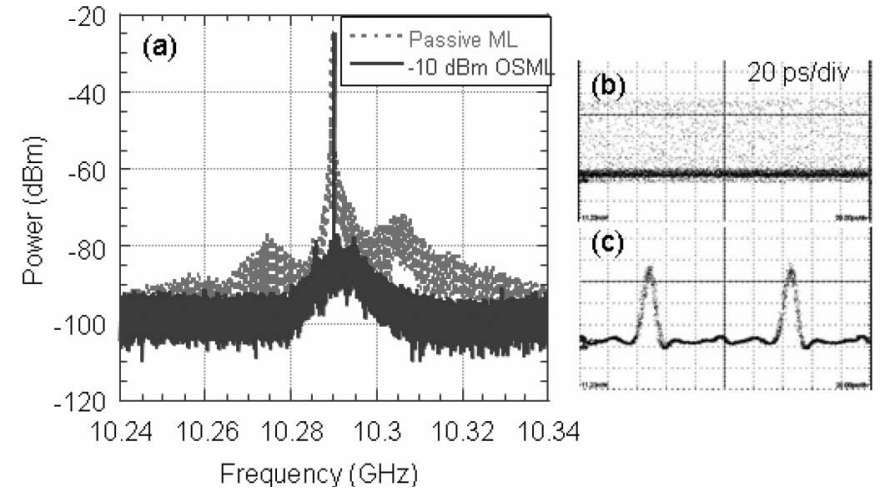

Fig. 7. (a) RF power spectra at the fundamental ML frequency of the $10-\mathrm{GHz}$ CPM laser under passive ML and optical injection locking conditions. Also, sampling scope traces of the CPM laser output triggered with the RF clock source under (a) passive (b) optical injection locking conditions.

sections and the SA pulse truncating effects, respectively [15]. Minimizing the active-passive interfacial reflections also plays an important role in achieving optimal pulse quality.

For synchronizing remote photonic-chip-based system nodes in an O-CDMA network, the electrical HML approach, while relatively easy to implement on a single device, requires supplying system clock signals at relatively high RF power levels at multiple distant locations, and is relatively cumbersome to implement. The alternative OSML approach would involve the simple routing of a single optical clock signal through the network fiber for injection locking of all CPM laser sources simultaneously.

For OSML optical injection locking characterization, a commercial tunable mode-locked fiber laser synchronized to an RF synthesizer clock source provides the optical clock, which is coupled into the $10-\mathrm{GHz}$ CPM laser through a polarizationmaintaining (PM) attenuator and a lensed fiber. The CPM output, collected through a lensed fiber from the other output facet, passes through a 3-nm tunable filter for removing any residuals of the injection signal, and then routes to different instruments for time- and frequency-domain measurements, and the bit-error-rate tester (BERT) for BER measurements.

Fig. 7(a) shows the RF power spectra of a $10-\mathrm{GHz}$ CPM laser under both passive ML and injection locking conditions at $-10 \mathrm{dBm}$ coupled optical power, with the injection signal at $1546 \mathrm{~nm}, 8 \mathrm{~nm}$ negatively detuned from the passive ML wavelength. Fig. 7(b) shows the corresponding 50-GHz optical sampling scope traces, triggered with the RF synthesizer clock. Compared to passive mode-locking, the RF spectral lineshape narrows significantly in Fig. 7(a) under optical injection, corresponding to the sharply reduced timing jitter after synchronization. The estimated root mean square (rms) timing jitter of the injection-locked CPM laser is 0.5 ps by integrating the singleside-band (SSB) phase noise spectrum, while the injected laser clock signal has an rms jitter of 0.17 ps. The effect of optical injection is primarily phase synchronization, without any observed changes in the pulse width and spectrum of the CPM laser output. Optical phase synchronization effects can be observed at injection power level as low as $-23 \mathrm{dBm}$. The BER measurements also demonstrated that the CPM output is error-free 


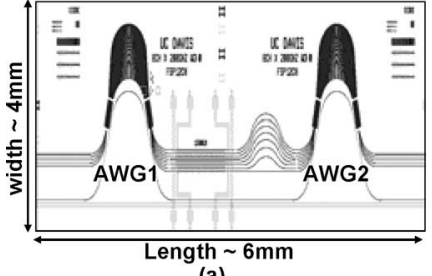

(a)

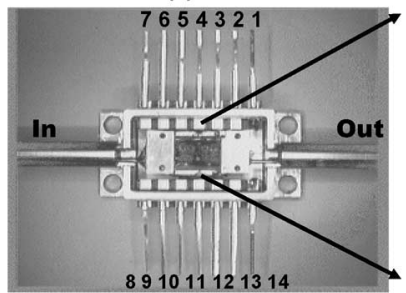

(c)

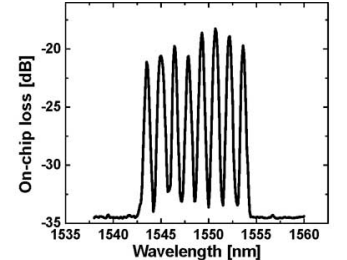

(b)

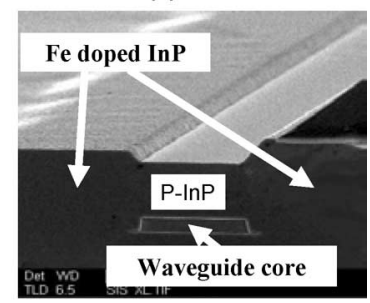

(d)
Fig. 8. (a) O-CDMA encoder chip layout, (b) encoder transmission spectrum, (c) packaged O-CDMA encoder chip, and (d) SEM picture of a laterally regrown passive waveguide.

with BER $<10^{-11}$ under $2^{23}-1$ pseudorandom bit sequence (PRBS) data modulation, demonstrating that an optical injection locked $10-\mathrm{GHz}$ CPM laser can indeed serve as a viable shortpulse light source in O-CDMA and other photonic integrated circuit applications.

\section{B. InP Encoder and Decoder}

The previous integrated O-CDMA encoders are based on either silica arrayed-waveguide gratings (AWG) with nonprogrammable external phase shifters [16] or on ring microresonators with relatively slow heater-based phase shifters [17]. On the other hand, InP platform-based SPECTS O-CDMA (Fig. 1) encoders and decoders offer rapid code reconfiguration by employing electrooptical phase shifters and the possibility of realizing monolithically integrated O-CDMA transmitters and receivers [5].

Fig. 8(a) shows the O-CDMA encoder mask layout. The AWG pair performs spectral demultiplexing and multiplexing. Phase modulators [cross section in Fig. 2(b)] in between the AWGs apply a phase shift, corresponding to the desired O-CDMA code, to each demultiplexed spectral channel. The input and output waveguides of the device are selected for optimal wavelength match of the two AWGs. Fig. 8(b) shows the transmission spectrum of an O-CDMA encoder with 32-dB fiber-to-fiber insertion loss. The loss can still be greatly reduced by improving the fiber coupling (here, 8-dB per fiber-to-chip coupling) and the fabrication process. Fig. 8(c) and (d) show the packaged chip and a scanning electron micrograph (SEM) picture of the cross section of the chip.

One of the challenges is to calibrate the phase response of the individual channels of the InP chip for two reasons. First, the phase-shifter response must be known as a function of injection current or reverse-bias voltage (both can be used to generate a phase shift) to apply the right amount of phase shift. Although phase shifter performance can be extracted using test structures, data from the actual shifters in the encoder is preferable due to

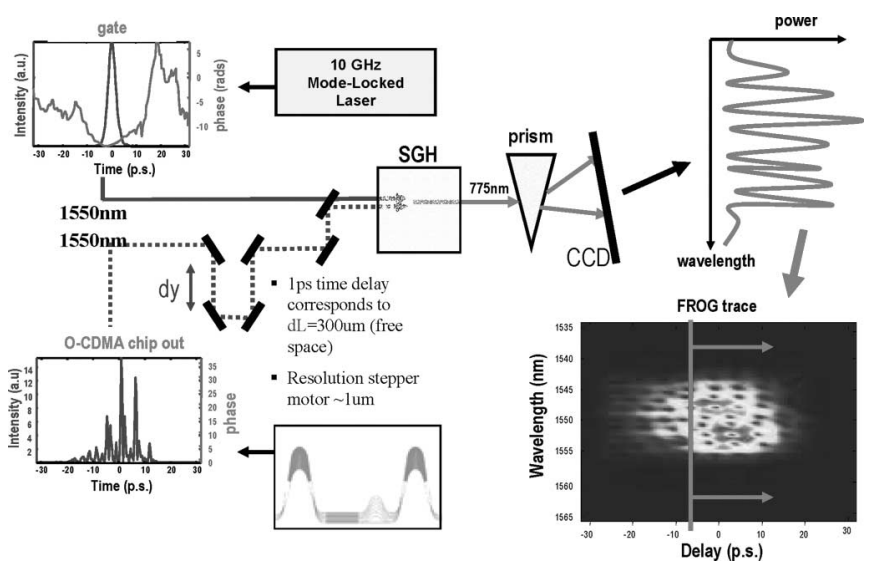

Fig. 9. Setup for XFROG-based phase-error measurement.

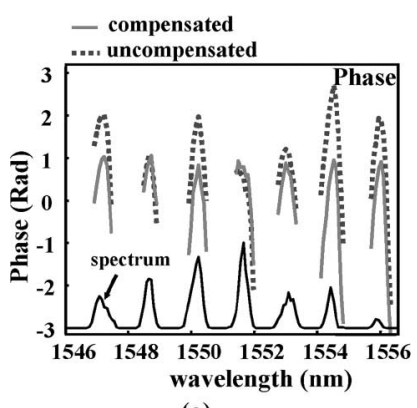

(a)

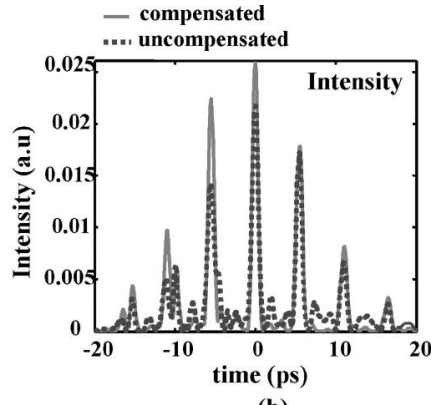

(b)
Fig. 10. (a) Phase and (b) intensity response of phase-compensated encoder.

possible variations between shifters. Second, the chip contains phase errors in the eight channels due to fabrication tolerances, causing a phase offset. For channel spacings up to $\sim 40 \mathrm{GHz}$ (note the encoder chip has $180 \mathrm{GHz}$ ), the differential phase of the channels can be extracted by detecting the coherent beating between two spectral channels of the encoder [18]. This approach requires the precise alignment of two axial modes from a mode-locked laser signal with two encoder channels. The two modes produce a coherent beating response at the encoder output. However, this beating is hard to detect beyond $40 \mathrm{GHz}$ without sophisticated high-speed electronics. In contrast, we characterized the phase response of the $180-\mathrm{GHz}$ encoder chip using the X-FROG technique [19], which avoids the precision spectral alignment requirements in the coherent beating approach. Also, the phase information from all channels is acquired in a single measurement.

Fig. 9 shows the principle of X-FROG-based phase-error measurement. The ML laser and compressor generated 0.5-ps pulses with a repetition rate of $10 \mathrm{GHz}$. A polarization controller (PC) put the pulses in TE polarization before a lensed fiber injected them into the encoder chip at $10 \mathrm{dBm}$. At the chip output, an erbium-doped fiber amplifier (EDFA) amplified the output pulse and fed it into the X-FROG through a PC. The amplified uncompressed pulse of the mode-locked laser served as reference for the X-FROG.

Fig. 10(a) shows the retrieved phase information (curve at the bottom corresponds to power in the channels), and Fig. 10(b) 

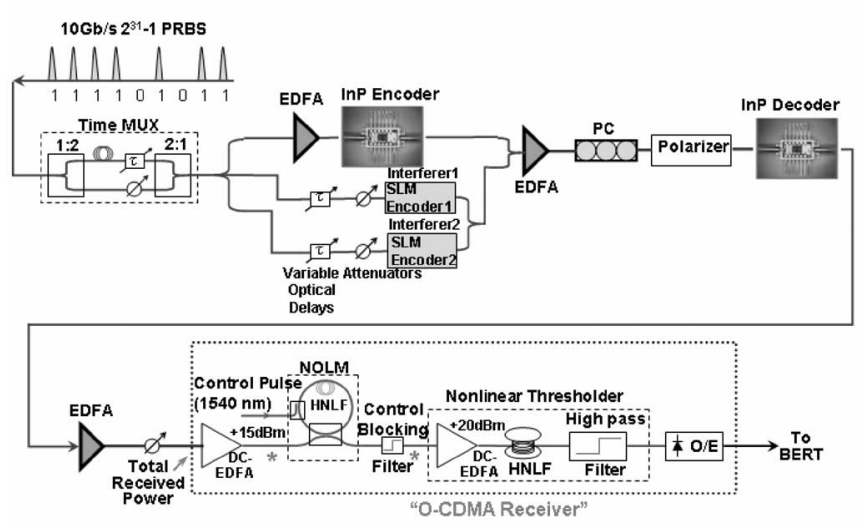

Fig. 11. Experimental setup for $6 \times 10 \mathrm{~Gb} / \mathrm{s}$ O-CDMA testbed.

shows the encoder output. The dotted lines in Fig. 10(a) and (b) are without phase-error compensation, solid lines with (by reverse-biasing the phase shifters). After compensation, the phases of all channels (peak of solid lines) are almost flat [Fig. 10(a)]. The compensation clearly improves the peak power and signal-to-noise (SNR) ratio [Fig. 10(b)]. The ringing peaks at 5.5-ps spacing originate from the $180-\mathrm{GHz}$ comb-shaped spectral response of the AWG [Fig. 8(b)]. This phase compensation result shows both the proper operation of the InP encoder and the value of FROG as encoder characterization tool.

Fig. 11 shows the schematic of the O-CDMA testbed. The mode-locked fiber laser source generated a 500 -fs pulse train at a $10-\mathrm{GHz}$ repetition rate. $\mathrm{A} \mathrm{LiNbO}_{3}$ modulator modulated the pulse train with a $10 \mathrm{~Gb} / \mathrm{s} 2^{31}-1$ PRBS pattern. A time-mux splitted the modulated train into two time slots of $50 \mathrm{ps}$ each. A dispersion-compensated erbium-doped fiber amplifier (DCEDFA) boosted the train, which is subsequently spatially split into three branches. One branch passes through the InP encoder [20] as the intended user (user channel). The other two branches are encoded by spatial light modulator (SLM) encoders [6], which acted as independent interferers (IF1 and IF2). The SLM encoders contained variable time delays for synchronization. After encoding, the intended user and interferers were combined and routed to the InP decoder. Upstream to each chip, an EDFA followed by a polarization controller supplied $21 \mathrm{dBm}$ of TE polarized light.

The InP devices each consist of an identical pair of 8-channel AWGs with $180-\mathrm{GHz}$ channel spacing and a free-spectral range (FSR) of 12-channel spacings. The 1.4-THz wavelength span provided by the eight channels is sufficient for encoding subpicosecond ( $\sim 0.5 \mathrm{ps})$ pulses. The InP devices are packaged in a standard 14-pin butterfly package with temperature control, which allows for an exact wavelength match of the encoder and decoder. The 2000- $\mu \mathrm{m}$-long phase-shifters are controlled by electrically biasing wire-bonded electrical leads.

We selected the 8-chip Walsh code [21] set for our spectral phase-coding experiment. The InP encoder applies code W1 containing the bits[11111111], whereas the InP decoder applies conjugate code $(*) \mathrm{W} 1 *$ [00000000]. Here, 1 denotes $\pi$ radians phase shift and 0 denotes zero phase shift in the respective phase
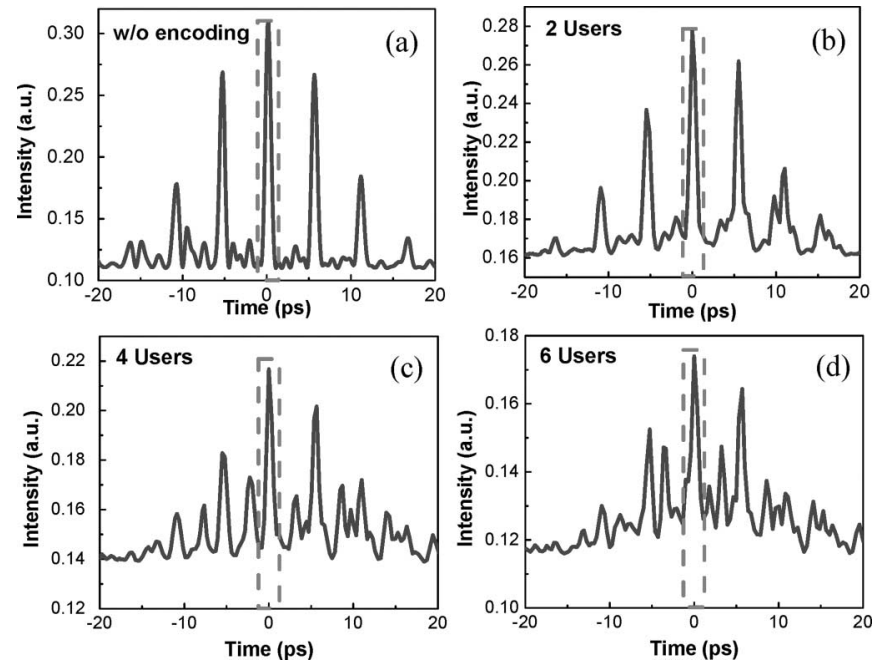

Fig. 12. Time-domain cross-correlation traces of the correctly decoded signal for (a) without coding, (b) 2 users, (c) 4 users and (d) 6 users.

shifters. SLM interferers IF1 and IF2 apply W2 [10101010] and W4 [10011001], respectively. These codes were selected for optimal suppression of the multiuser interference (MUI). The gate [a nonlinear optical loop mirror (NOLM)], to be replaced by the integrated MZI, is followed by power thresholding with highly nonlinear fiber (HNLF) and a long-pass filter. This combination suppresses an incorrectly decoded signal while it passes a correctly decoded signal.

Fig. 12 displays the effect of MUI on the pulse output. Fig. 12(a) shows the cross-correlation trace after the encoderdecoder pair with phase-error compensation but without coding. The $\sim 3$-ps time gating around $t=0 \mathrm{ps}$ (dotted line) removes the ringing peaks at the expense of signal power. Fig. 12(b) shows the cross-correlation trace of the output of the encoder-decoder pair without interferers (extra users), with $\mathrm{W} 1$ and $\mathrm{W} 1 *$ codes for encoder and decoder for two time slots. Fig. 12(c) and (d) show the traces with added interferer W2 and interferers W2 and $\mathrm{W} 4$, respectively (two time slots).

Fig. 13(a) shows the BER measurement results for the 6-user O-CDMA system with the InP encoder and decoder. Back-toback data were taken with the O-CDMA encoder and decoder pair bypassed. The received power in all BER curves is defined as the average input power per user as measured at the input of the "O-CDMA receiver." The 2-user and 4-user experiments demonstrated error-free operation and a power penalty of $5.5 \mathrm{~dB}$ at $\mathrm{BER}=10^{-9}$ with respect to back-to-back data. The penalty can be mainly attributed to the power lost to the ringing peaks. The 6-user case attained an error floor at $10^{-9}$, primarily due to MUI inside the time gate. The MUI effects are relatively small for 2 and 4 users, resulting in nonobservable noise floor. For a narrower time gate, window and/or longer code length would mitigate the MUI effects significantly and an error-free operation is expected.

In order to investigate the effect of the spectral filtering and loss in the InP-based en/decoders, we repeated the BER measurement but replaced the InP devices with SLMbased en/decoders. The SLM devices approach an ideal coding 


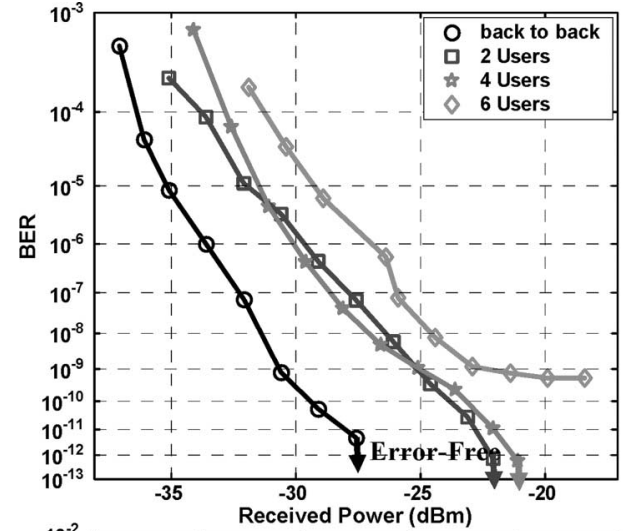

(a)

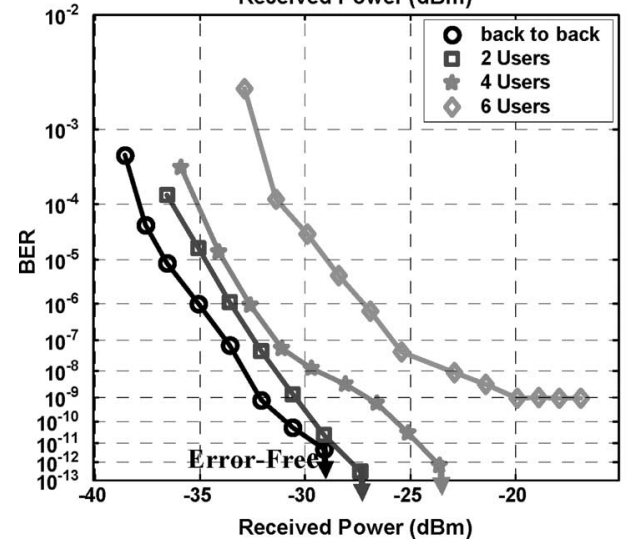

(b)

Fig. 13. BER measurement results for (a) InP encoder and decoder chips and (b) SLM encoders and decoder.

performance due to their rectangular filter shape. Fig. 13(b) shows the BER measurement result and eye diagrams. The 2-user and 4-user experiments demonstrated error-free operation with a power penalty of 2 and $5 \mathrm{~dB}$, respectively, at $\mathrm{BER}=10^{-9}$. The error floor below $10^{-9}$ in the 6-user SLM case confirms that MUI, and not the InP chip response, is the dominant limitation for error-free operation of the O-CDMA system.

\section{MZI Threshold Detector Development}

The last step of the SPECT O-CDMA transmission link requires nonlinear optical thresholding: the detection of properly decoded optical pulses with narrow pulse width and high peak power, and the blocking of the time spread by improperly decoded interferer signal. Compared to other nonlinear-fiber-based thresholder approaches, the MZI thresholder is less bulky and more sensitive [22], [23] and is compatible with monolithic integration with other semiconductor components. Here, we describe an improved "clock-gated" MZI scheme with respect to the "self-gated" approach reported previously [5]. Both schemes depend on differential operation of the MZI.

Fig. 14 shows the self-gated and clock-gated MZI operation. In the self-gated scheme, no external clock signal is provided. The decoded pulse is split into two, and each part enters a distinct MZI arm with a time delay. Only the correctly decoded pulses have sufficiently fast rising edges to saturate the nonlinear optical elements in the MZI arms, opening a differential gate for

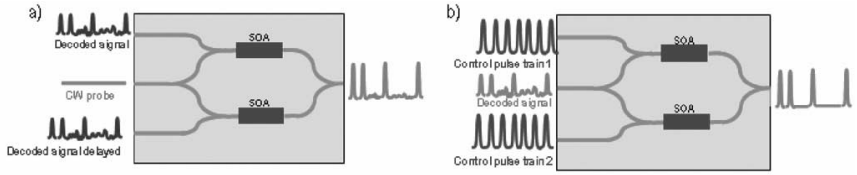

Fig. 14. O-CDMA thresholder in (a) nongated and (b) clock-gated configuration.

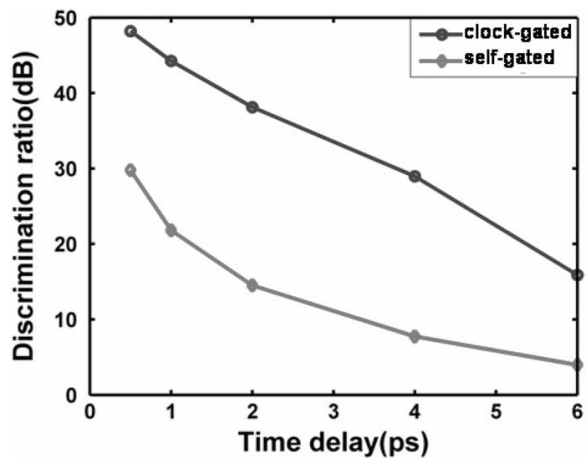

Fig. 15. Graph of power ratio after MZI thresholder for self-gated and clockgated operations.

the CW probe signal. The clock-gated scheme uses the external short-pulse clock signal for generating the differential gate of the MZI.

Fig. 15 shows the simulated discrimination ratio (the ratio of the integrated energy of a correctly decoded pulse versus that of an incorrectly decoded pulse) as a function of the time delay between the two MZI arms for self-gated and clock-gated operation using 64-bit Walsh codes. Clock-gating outperforms self-gating by up to $20 \mathrm{~dB}$, because in the self-gated condition, a partial broad gate can still be present for incorrectly decoded signal, resulting in incomplete blocking.

For improved performance, we investigated a new class of integrated MZI based on reverse-biased EA [24]. The reversebiased EA sections result in faster carrier sweepout (tens of picoseconds) and less amplified stimulated emission (ASE) noise compared to the conventional forward-biased SOAs in the MZI arms favoring high speed and lower power consumption operation with greater cascadability.

Fig. 16(a) shows the fabricated EA-MZI with four multimode interference (MMI)-based 3-dB couplers, two active EA sections of 200 and $50 \mu \mathrm{m}$ in length, and two 600- $\mu \mathrm{m}$-long phase shifters (see also Fig. 2). The phase modulators allow the MZI to be biased for optimal destructive interference at the MZI output, whereas the unequal EA section lengths allow better power balance between the two arms (without pump). Injection of an optical pump signal into the shortest EA induces cross phase and amplitude modulation, and the device operates as a "noninverting" optical switch (or wavelength converter).

Fig. 16(b) shows the normalized MZI switching output versus pump input power with the pump signal at $1540 \mathrm{~nm}$, probe signal at $1550 \mathrm{~nm}$, and the upper and lower EA reverse biased at -1 and $-12 \mathrm{~V}$, respectively. A 10-dB pump power change (ER) resulted in 18-dB output switching ratio, demonstrating the optical signal regeneration capability of the EA-MZI. Fig. 16(b) 


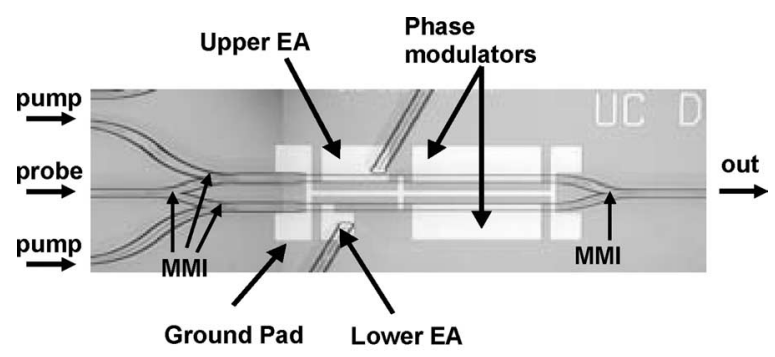

(a)

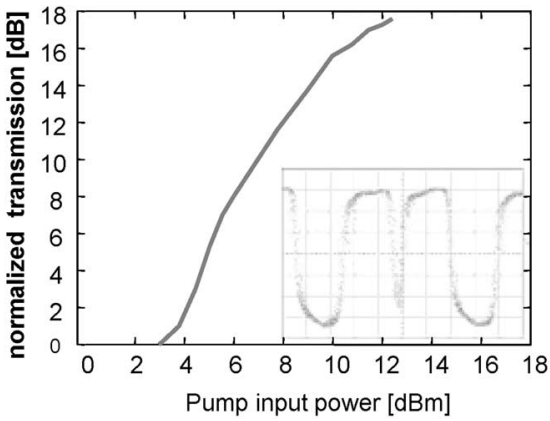

(b)

Fig. 16. (a) Photograph of fabricated EA-MZI with active-passive integrated waveguide. b) Normalized MZI transmission versus pump input power, inset showing EA-MZI output at $2.5 \mathrm{~Gb} / \mathrm{s}$.

inset shows the MZI wavelength conversion output from a $2.5 \mathrm{~Gb} / \mathrm{s} 2^{7}-1$ PRBS pump input. These results demonstrate the optical switching capacity of the EA-MZI device, and its potential as a high-speed, low-power, and low-noise optical thresholder element.

\section{FUTURE WORK}

It is a challenging task to achieve a monolithically integrated on-chip system. To make the on-chip O-CDMA system operate properly, several key issues need to be addressed. These include low-loss waveguide, the realization of narrow channel spacing AWG, on-chip mirror for lasing operation of the mode-locked laser. By optimizing the layer stack of the material system of the device and processing procedure of the fabrication, we achieved $3 \mathrm{~dB} / \mathrm{cm}$ for passive waveguide for doped material. This lowloss waveguide combined with on-chip amplification by using SOA can satisfy the requirement for integrated system. We will discuss two other issues in this section.

\section{A. Realization of Narrow Channel Count AWG}

For O-CDMA, the amount of users can be increased if the (AWG) channel spacing is reduced or if the bandwidth of the laser pulses is increased. The narrower the channel spacing, the longer the code length that can be applied to allow better suppression of MUI [5]. Currently, we are working toward 50 and $20 \mathrm{GHz}$ channel spacing encoders. As an intermediate step, we have developed a $100-\mathrm{GHz}$ channel spacing AWG to optimize the layer stack, fabrication the processing step and AWG design parameters (such as input/output free-propagation region and waveguide shape).

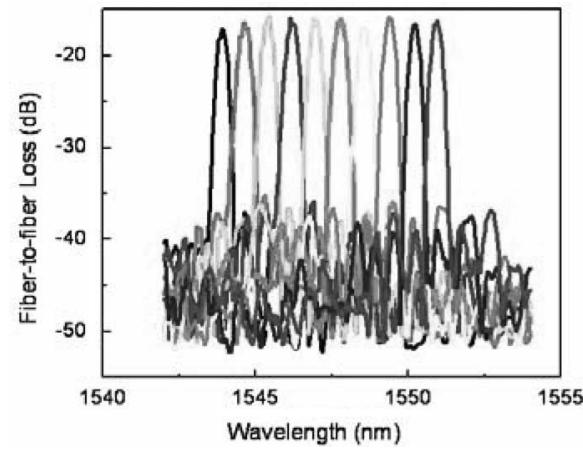

Fig. 17. Transmission spectra of all ten transmission channels of $100-\mathrm{GHz}$ channel spacing AWG.

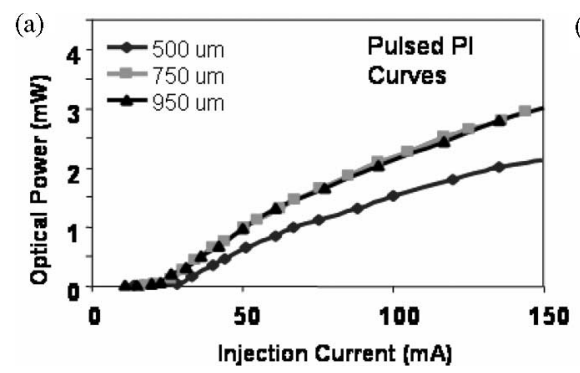

(b)

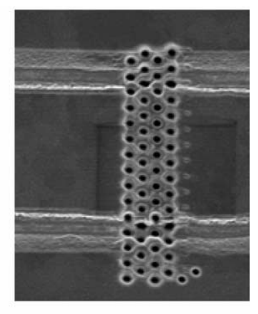

Fig. 18. (a) Optical output of etched facet laser versus injection current (1\% duty cycle) and (b) FIB-etched photonic crystal laser mirror.

Fig. 17 shows the transmission spectrum of a 10 channel $\times$ $100 \mathrm{GHz}$ channel spacing AWG. The FSR of this AWG is $12.8 \mathrm{~nm}$, which is the same as our 8-channel device. The $3-\mathrm{dB}$ bandwidth of single channel is $0.35 \mathrm{~nm}$. The fiber-to-fiber loss of this single AWG is $16 \mathrm{~dB}$. The SNR is more than $25 \mathrm{~dB}$, and side-lobe suppression is about $20 \mathrm{~dB}$. The channel spacing is $0.8 \mathrm{~nm}$. The central wavelength (channel 6) is $1547.7 \mathrm{~nm}$. The nonuniformity among 10 channels is less than $2 \mathrm{~dB}$. Compared to 8-channel $\times 200 \mathrm{GHz}$ channel spacing device, the fiberto-fiber loss of the $100-\mathrm{GHz}$ design does not increase. This proves that the improved design allows narrower channel spacings without performance loss, making higher channel count AWGs feasible. Furthermore, we have characterized the phase errors in the AWG arms using a new method: XFROG in combination with an inverse Fourier transform. Knowledge of these errors will enable us to reduce systematic phase errors in the AWG arms.

\section{B. On-Chip Mirror for Mode-Locked Laser Integration}

Monolithic integration of the CPM laser source with other elements requires the development of built-in mirror reflectors [Fig. 2(d)]. We are developing the photonic crystal (PC)-based laser mirrors similar to that demonstrated on $\mathrm{CW}$ semiconductor lasers [25]. The etched mirrors allow for accurate control of the repetition rate. Fig. 18(b) shows the photonic crystal mirrors fabricated using focused-ion-beam (FIB) and titanium etch mask. Fig. 18(a) displays pulsed laser output for three laser cavities having different lengths. Lasing threshold currents observed were around $25 \mathrm{~mA}$ (1\% duty cycle). The analyses of the Fourier 
components of the output spectra confirmed lasing action in the cavity defined by the etched mirrors instead of another possible cavity defined between the antireflection-coated cleaved facets.

\section{CONCLUSION}

InP platforms have strong potentials to provide compact and stable monolithic integration for system scale applications. We presented two InP integration platforms and demonstrated up to $60 \mathrm{~Gb} / \mathrm{s}$ O-CDMA using InP encoders/decoders. Adding our results on the $10 \mathrm{~Gb} / \mathrm{s}$ mode-locked laser transmitter with nearly transform-limited 1.75-ps pulses and the EA-based MZI threshold detector, we firmly believe in the feasibility of a single O-CDMA transceiver chip with transmission capacity beyond $100 \mathrm{~Gb} / \mathrm{s}$. Future work involves improved channel count by denser spectral resolution of the O-CDMA system and shorter mode-locked laser pulses for wider pulse spectra.

\section{REFERENCES}

[1] E. J. Skogen, J. W. Raring, G. B. Morrison, C. S. Wang, V. Lal, M. L. Masanovic, and L. A. Coldren, "Monolithically integrated active components: A quantum-well intermixing approach," IEEE J. Sel. Top. Quantum Electron., vol. 11, no. 2, pp. 343-355, Mar./Apr. 2005.

[2] J. J. M. Binsma, J. H. den Besten, and R. G. Broeke, "InP-based photonic integration technology," presented at the Opt. Amplifiers Their Appl./Integr. Photon. Res. Topical Meet., 2004, Paper IFB1.

[3] E. Bente and M. Smit, "Ultra fast InP integrated circuits," Proc. SPIE, vol. 6124, pp. 612419-612444, 2006.

[4] S. R. Forrest, M. R. Gokhale, P. V. Studenkov, and F. Xia, "Integrated photonics using asymmetric twin-waveguide structures," in Proc. Int. Conf. Indium Phosphide Related Mater., 2000, pp. 13-16.

[5] C. Ji, R. G. Broeke, Y. Du, J. Cao, N. Chubun, P. Bjeletich, F. Olsson, S. Lourdudoss, R. Welty, C. Reinhardt, P. L. Stephan, and S. J. B. Yoo, "Monolithically integrated InP based photonic chip development for OCDMA systems," IEEE J. Sel. Top. Quantum Electron., vol. 11, no. 1, pp. 66-77, Jan./Feb. 2005.

[6] V. J. Hernandez, W. Cong, J. Hu, C. Yang, N. K. Fontaine, R. P. Scott, Z. Ding, B. H. Kolner, J. P. Heritage, and S. J. B. Yoo, "A 320-Gb/s capacity $(32$-User $\times 10 \mathrm{~Gb} / \mathrm{s})$ SPECTS O-CDMA network testbed with enhanced spectral efficiency through forward error correction," J. Lightw. Technol., vol. 25, no. 1, pp. 79-86, Jan. 2007.

[7] R. P. Scott, N. K. Fontaine, J. P. Heritage, B. H. Kolner, and S. J. B. Yoo, "3.5-THz wide, 175 mode optical comb source," presented at the IEEE/OSA Opt. Fiber Commun. Conf., 2007, Paper OWJ3.

[8] C. Ji, N. Chubun, R. G. Broeke, J. Cao, Y. Du, S. J. B. Yoo, K. Y. Liou, J. R. Lothian, S. Vatanapradit, S. N. G. Chu, B. Patel, W. S. Hobson, and W. T. Tsang, "Synchronized transform-limited operation of $10-\mathrm{GHz}$ colliding pulse mode-locked laser," IEEE Photon. Technol. Lett., vol. 18, no. 4, pp. 625-627, Feb. 2006.

[9] Y. K. Chen and M. C. Wu, "Monolithic colliding-pulse mode-locked quantum-well lasers," IEEE J. Quantum Electron., vol. 28, no. 10, pp. 2176-2185, Oct. 1992.

[10] K. Yvind, D. Larsson, L. J. Christiansen, J. Mork, J. M. Hvam, and J. Hanberg, "High-performance $10 \mathrm{GHz}$ all-active monolithic modelocked semiconductor lasers," Electron. Lett., vol. 40, no. 12, pp. 735-737, Jun. 2004.

[11] H. Fan, C. Wu, M. El-Aasser, N. K. Dutta, U. Koren, and A. B. Piccirilli, "Colliding pulse mode-locked laser," IEEE Photon. Technol. Lett., vol. 12, no. 8, pp. 972-973, Aug. 2000.

[12] S. Arahira and Y. Ogawa, "480-GHz subharmonic synchronous mode locking in a short-cavity colliding-pulse mode-locked laser diode," IEEE Photon. Technol. Lett., vol. 14, no. 4, pp. 537-539, Apr. 2002.

[13] H. Kurita, T. Shimizu, and H. Yokoyama, "Experimental investigations of harmonic synchronization conditions and mechanisms of mode locked laser diodes by optical pulse injection," IEEE J. Sel. Top. Quantum Electron., vol. 2, no. 3, pp. 508-513, Sep. 1996.

[14] R. Trebino, Frequency Resolved Optical Gating: The Measurement Of Ultrashort Laser Pulses. Boston, MA: Kluwer, 2000.
[15] S. Gee, R. Coffie, P. J. Delfyett, G. Alphonse, and J. Connolly, "Intracavity gain and absorption dynamics of hybrid modelocked semiconductor lasers using multiple quantum well saturable absorbers," Appl. Phys. Lett., vol. 71, pp. 2569-2571, 1997.

[16] H. Tsuda, H. Takenouchi, T. Ishii, K. Okamoto, T. Goh, K. Sato, A. Hirano, T. Kurokawa, and C. Amano, "Spectral encoding and decoding of $10 \mathrm{Gbit} / \mathrm{s}$ femtosecond pulses using high resolution arrayed-waveguide grating," Electron. Lett., vol. 35, no. 14, pp. 1186-1188, Jul. 1999.

[17] A. Agarwal, P. Toliver, R. Menendez, S. Etemad, J. Jackel, J. Young, T. Banwell, B. E. Little, S. T. Chu, W. Chen, W. Chen, J. Hryniewicz, F. Johnson, D. Gill, O. King, R. Davidson, K. Donovan, and P. J. Delfyett, "Fully programmable ring-resonator-based integrated photonic circuit for phase coherent applications," IEEE J. Lightw. Technol., vol. 24, no. 1, pp. 77-87, Jan. 2006.

[18] A. Agarwal, P. Toliver, R. Menendez, S. Etemad, J. Jackel, J. Young, T. Banwell, B. E. Little, S. T. Chu, and P. Delfyett, "Fully-programmable ring resonator based integrated photonic circuit for phase coherent applications," presented at OFC, 2005, Paper PDP6.

[19] R. G. Broeke, J. Cao, N. K. Fontaine, C. Ji, N. Chubun, F. Olsson S. Lourdudoss, B. H. Kolner, J. P. Heritage, and S. J. B. Yoo, "Phase characterization of an inp based optical-cdma encoder using frequencyresolved optical gating (FROG)," presented at the IEEE LEOS Annu. Meet., 2005, Paper ML2.

[20] J. Cao, R. G. Broeke, N. Fontaine, C. Ji, Y. Du, N. Chubun, K. Aihara, A.-V. Pham, F. Olsson, S. Lourdudoss, and S. J. B. Yoo, "Demonstration of spectral phase O-CDMA encoding and decoding in monolithically integrated arrayed-waveguide-grating-based encoder," IEEE Photon. Technol. Lett., 2007, to be published.

[21] F. J. MacWilliams and N. J. A. Sloane, The Theory of Error Correcting Codes. New York: North-Holland, 1977.

[22] H. P. Sardesai and A. M. Weiner, "Nonlinear fibre-optic receiver for ultrashort pulse code division multiple access communications," Electron. Lett., vol. 33, no. 7, pp. 610-611, Mar. 1997.

[23] Z. Jiang et al., "Spectrally coded O-CDMA system with four users at 2.5 Gbit/s using low power nonlinear processing," Electron. Lett., vol. 40, no. 10, pp. 623-625, May 2004.

[24] M. Suzuki, H. Tanaka, and S. Akiba, "Effect of hole pileup at heterointerface of modulation voltage in GaInAsP electroabsorption modulators," Electron. Lett., vol. 25, no. 2, pp. 88-89, Jan. 1989.

[25] J. Moosburger, M. Kamp, F. Klopf, J. P. Reithmaier, and A. Forchel "Semiconductor lasers with 2D photonic crystal mirrors based on a wetoxided Al2O3-mask," IEEE Photon.Technol.Lett., vol. 13, no. 5, pp. 406408, May 2001.

Ronald G. Broeke received the M.S. degree in physics from the University of Utrecht, Utrecht, The Netherlands, in 1998, and the Ph.D. degree from the Department of Electrical Engineering, Delft University of Technology, Delft, The Netherlands in 2003.

He is currently a Postdoctoral Research Scientist with the Department of Electrical and Computer Engineering, University of California, Davis.

Jing Cao (S'01) received the B.S. and M.S degrees from the Department of Electronics Engineering, Tsinghua University, Beijing, China in 1997 and 2000, respectively. He is currently working toward the Graduate degree at the Department of Electrical and Computer Engineering, University of California, Davis.

His current research interests include optical integrated devices and system integration for next generation optical network.

Mr. Cao is a member of the Optical Society of America.

Chen Ji received the B.S. degree in physics from the University of Illinois, Urbana-Campaign, Urbana, in 1993, and the Ph.D. degree in electrical engineering from Cornell University, Ithaca, NY, in 1999.

He is currently a Postdoctoral Research Scientist with the Department of Electrical and Computer Engineering, University of California, Davis. His research interests include semiconductor lasers, optical integrates devices, and system integration for next generation optical networks. 


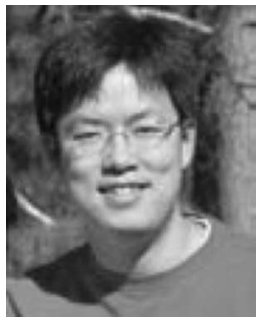

Sang-Woo Seo (M'03) received the B.S. degree in electrical and computer engineering from Ajou University, Suwon, Korea, in 1997, the M.S degree in information and communications from Kwangju Institute of Science and Technology (KJIST), Kwangju, Korea, in 1999, and the Ph.D. degree in electrical and computer engineering from Georgia Institute of Technology, Atlanta, in 2003.

From 2003 to 2005, he was a Research Engineer with Georgia Institute of Technology and Duke University, Durham, NC. Since 2005, he has been with the University of California, Davis. His current interests include high-speed modulators, photonic integrated devices, and system integration for optical code division access network and optical arbitrary waveform generation.

Yixue Du (S'03) received the M.S. degree in physics from Oklahoma State University, Stillwater, OK, in 1998. She is currently working toward the Ph.D. degree in electrical engineering at the University of California, Davis.

Her research interests include simulation of the network behavior, and the design and testing of nonlinear optical thresholding device in optical code division multiple access networks.

Nick K. Fontaine received the B.S. degree in electrical engineering and optical engineering from the University of California, Davis, in 2004, where he is currently working toward the Ph.D. degree.

His research interests include frequency resolved optical gating, optical arbitrary waveform generation, optical code-division multiple access, and very small lasers.

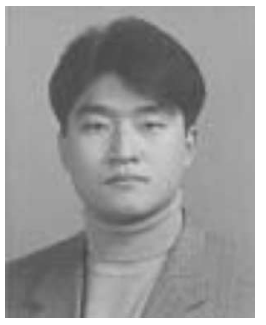

Jong-Hwa Baek received the M.S. and Ph.D. degrees in physics from Korea Advanced Institute of Science and Technology, Daejeon, Korea, in 1999 and 2004, respectively.

He is currently a Research Scientist with the University of California, Davis. His research interests include photonic integrated circuits, photonic crystal active devices, and nanophotonic technologies.

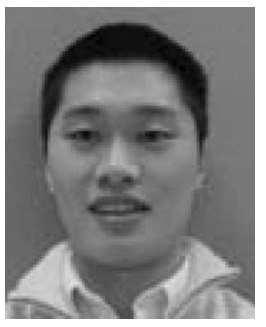

John Yan received the B.S. degree in electrical engineering from the University of California, Davis, in 2005 , where he is currently working toward the Ph.D. degree.

His research interests include optical semiconductor integration, radio-frequency ICs and mixed-signal ICs.

Mr. Yan is a member of the Tau Beta Pi.

Francisco M. Soares received the M.S. degree in electrical engineering from the Delft University of Technology, The Netherlands, in 2000. In 2006, he received the Ph.D. degree from the Department of Electrical Engineering, Technical University of Eindhoven, Eindhoven, The Netherlands.

Since 2006, he is with the University of California, Davis, where he is engaged in research on optical arbitrary waveform generators in InP technology. His research interests include theories, modeling, characterization, and fabrication of compound semiconductor devices such as optical switches and modulators, arrayed waveguide gratings, spot-size converters, and multimode interference couplers.
Fredrik Olsson received the B.S. degree in physics from the Royal Institute of Technology (KTH), Stockholm, Sweden, in 2000, where he is currently working toward the Ph.D. degree at the School of Information and Communication Technology.

His research interests include integration of III/V semiconductor materials on silicon and growth of optical integrated devices.

S. Lourdudoss (M'97-SM'99) received the M.S. degree in chemistry from Madras University, Chennai, India, in 1976 and the Ph.D. degree in chemistry from Faculté Libre des Sciences de Lille, Lille, France, in 1979.

He was a Postdoctoral Fellow at the Royal Institute of Technology (KTH), Stockholm, Sweden, where he worked on the thermochemical aspects of thermal energy storage and absorption heat pumps until 1985. He then joined the Swedish Institute of Microelectronics, Stockholm. Currently, he is a Professor of semiconductor materials at KTH, and is involved in undergraduate and graduate education. His research interests include MOVPE of GaN and related materials, and the issues related to the monolithic integration of III-V materials on a single platform as well as on Si by HVPE.

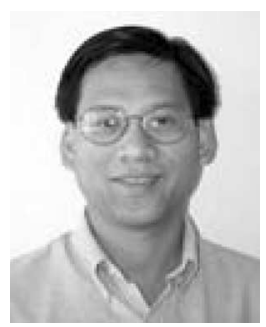

Anh-Vu H. Pham (SM'03) received the B.E.E. (with honors), M.S., and Ph.D. degrees from the Georgia Institute of Technology, Atlanta, in 1995, 1997, and 1999, respectively.

He cofounded RF Solutions, LLC, Warren, NJ, in 1997, which was acquired by Anadigics in 2003. He has held Faculty position with Clemson University, Clemson, SC. He is currently an Associate Professor with the University of California, Davis. He has coauthored about 80 technical papers published in several journals and conference proceedings. His research interests include RF and high-speed packaging and signal integrity, and RFIC design.

Prof. Pham serves as a member of the IEEE Microwave Theory and Techniques Society (IEEE MTT-S) International Microwave Symposium's Technical Program Committee on Power Amplifiers and Integrated Circuits. He has been the Chair of the IEEE MTT-12 Microwave and Millimeter Wave Packaging and Manufacturing Technical Committee of the IEEE MTT-S. He was the recipient of the 2001 National Science Foundation CAREER Award.

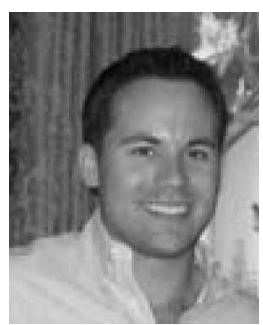

Michael Shearn received the B.S. degrees in electrical engineering, physics, and mathematics from Southern Methodist University, Dallas, TX, in 2004. $\mathrm{He}$ is currently working toward the Ph.D. degree in applied physics at the California Institute of Technology, Pasadena.

His research interests include silicon photonics, optical resonators, and novel fabrication techniques.

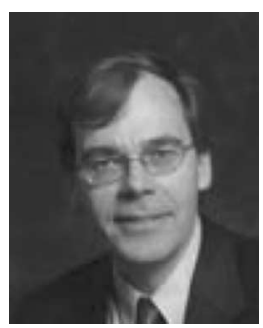

Axel Scherer received the B.S., M.S., and Ph.D. degrees from the New Mexico Institute of Mining and Technology, Socorro, New Mexico, in 1981, 1982, and 1985 , respectively.

From 1985 to 1993 , he was with the Quantum Device Fabrication Group, Bellcore, Morristown, NJ. $\mathrm{He}$ is currently the Bernard E. Neches Professor of Electrical Engineering, Applied Physics, and Physics with the California Institute of Technology, Pasadena, specializing in device microfabrication. His research interests include design and fabrication of functional photonic, nanomagnetic, and microfluidic devices. 


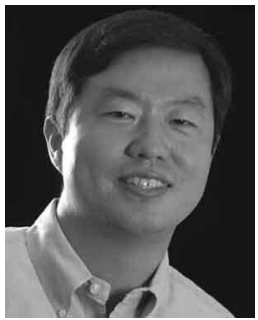

S. J. Ben Yoo (S'82-M'84-SM'97) received the B.S., M.S., and Ph.D. degrees in electrical engineering from Stanford University, Stanford, CA, in 1984, 1986, and 1991, respectively.

$\mathrm{He}$ is currently a Professor of electrical engineering with the University of California, Davis (UC Davis), where he is also the Director of Center for Information Technology Research in the Interest of Society. His research interests include high-performance all-optical devices, systems, and networking technologies for the next generation Internet; and architectures, systems integration, and network experiments related to all-optical label switching routers and optical code division multiple access technologies. Prior to joining UC Davis in 1999, he was a Senior Research Scientist with Bell Communications Research, Morristown, NJ, leading technical efforts in optical networking research and systems integration. He was with Stanford University, Stanford, CA, before joining Bell Communications Research. He also conducted research on lifetime measurements of intersubband transitions and on nonlinear optical storage mechanisms at Bell Laboratories, Murray Hill, NJ, and IBM Research Laboratories, San Jose, CA, respectively.

Prof. Yoo is a Fellow of the IEEE Lasers and Electro-Optics Society and a Fellow of the Optical Society of America. He is a member of the Tau Beta Pi. He was the recipient of the DARPA Award for Sustained Excellence in 1997, the Bellcore CEO Award in 1998, and the Mid-Career Research Faculty Award (UC Davis) in 2004. He was the Co-Chair of the Technical Program Committee for APOC 2004 and APOC 2003, and was a member of technical program committees for several conferences. He is the General Co-Chair for IEEE LEOS Photonics in Switching Conference 2007. He is an Associate Editor for IEEE Photonics Technology LetTers, and a Guest Editor for IEEE/OSA JourNAL OF LIGHTWAVE TECHNOLOGY in 2005 and IEEE JOURNAL OF SELECTED TOPICS IN QUANTUM ELECTRONICS in 2007. 